\title{
RF MEMS: Silicon micro-mechanical capacitive structures.
}

\author{
G. Bazin*, J.P. Gilles**, P. Crozat**, Souhil Megherbi** \\ *Groupe ESIEE - Noisy-le-Grand - France, **IEF - Université Paris Sud - Orsay - France \\ bazing@esiee.fr, jean-paul.gilles@ief.u-psud.fr, paul.crozat@ief.u-psud.fr,sm@ief.u-psud.fr
}

\begin{abstract}
The general study is dedicated to Wireless Sensor Networks. The idea is to associate micro-mechanical and microwave communication functions to exchange information between a remote sensor and a base station [1]. A specific part of this project concerns the conception, realisation, and characterisation of a passive microwave frequency shifter used for back-modulation around $2 \mathrm{GHz}$. The interesting point was to develop this function with an integrated silicon micro-mechanical rotating structure. The paper describes the prototype of the RF MEMS already developed in ESIEE laboratory. And we insist on the microwave measurements performed and the associated equivalent models.
\end{abstract}

Keywords - wireless sensor networks, RF MEMS, electrostatic actuation, microwave frequency shifter.

\section{INTRODUCTION AND PRINCIPLE}

The work is a part of a project in Wireless Sensor Networks, in connection with Thomson Detexis, Figure 1. At present time, we focused on the backmodulation function to collect and send back data from the remote sensor to the base station using a passive frequency shifter.

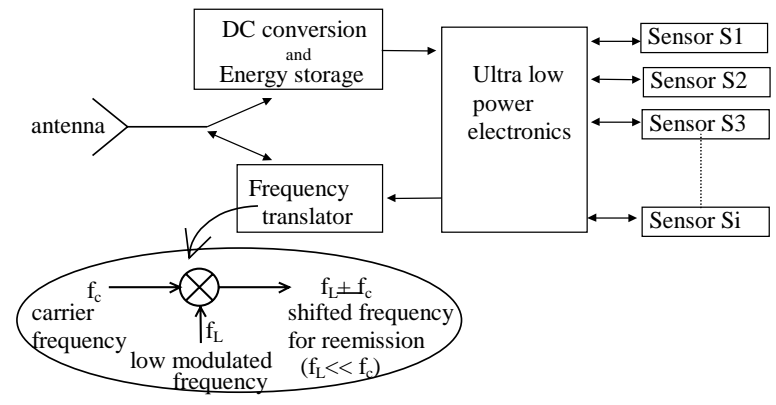

Figure 1. The Wireless Sensor Network context.

The microwave carrier $\mathrm{f}_{\mathrm{c}}$, around $2 \mathrm{GHz}$, is slightly translated after mixing a low frequency wave $\mathrm{fL}_{\mathrm{L}}$ between $1 \mathrm{kHz}$ and $50 \mathrm{kHz}$, modulated with the sensor data at a very low bit rate.
The originality is to perform mixing with RF MEMS integrated on silicon, compared to classical diode mixers. The frequency shifter is made of two coupled capacitors integrated on the same micro-system moving in opposition at the low frequency rate. Electrostatic actuation is used to control the gap between two electrodes, see Figure 2.

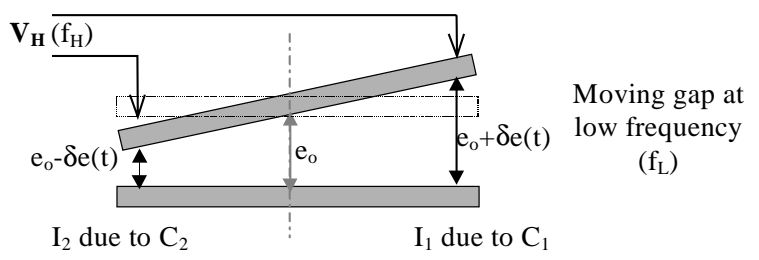

Figure 2. Principle of the micro-mechanical capacitors.

When connecting the two capacitors properly, see Figure 3, we collect a current I proportional to the product of the low frequency capacitance variations and the high frequency voltage time variations, which is the required mixing function [2].

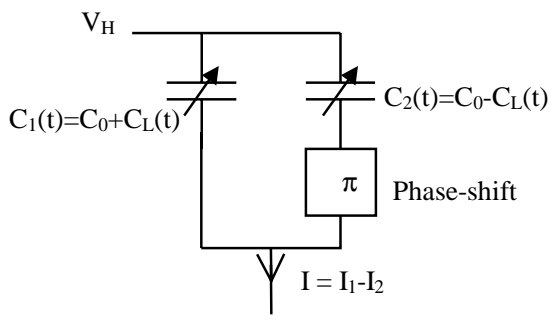

Figure 3. Differential measurement of the current.

\section{DESCRIPTION OF THE PROTOTYPE}

The device is made with a silicon substrate (SOI type) onto a glass substrate. In our case, the twocoupled micro-capacitors require a rotating structure like a torsional plate [3][4]. Electrodes $(\mathrm{Cr} / \mathrm{Au})$ on silicon are located in regard to the same metallisations on glass to create the moving capacitors, Figure 4. 


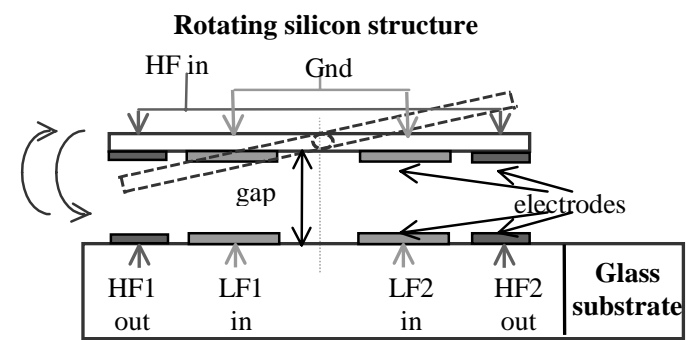

Figure 4. Electrodes on the rotating structure.

An electro-mechanical analytic model correlated with simulations has been made to optimise the dimensions of the device. We have selected for the geometry rectangle $(\mathrm{R})$ and square $(\mathrm{S})$ plates, as given in Table 1.

\begin{tabular}{|l|l|}
\hline geometrical description & dimension, $\boldsymbol{\mu m}$ \\
\hline total structure's length & $2500(\mathrm{R}), 1400(\mathrm{~S})$ \\
\hline total structure's width & $1400(\mathrm{R} \& \mathrm{~S})$ \\
\hline length of torsional arms & $400(\mathrm{R} \mathrm{\&} \mathrm{S})$ \\
\hline width of torsional arms & $185(\mathrm{R}), 100(\mathrm{~S})$ \\
\hline thickness of the arms & 15 \\
\hline air gap & 4 \\
\hline
\end{tabular}

Table 1. Dimensions of the prototype.

The HF capacitors at equilibrium are calculated to approach $1 \mathrm{pF}(\mathrm{R})$ and $0.7 \mathrm{pF}(\mathrm{S})$. The theoretical mechanical resonance frequency is around $10 \mathrm{kHz}$, and the maximum rotating angle is less than $5.10^{-4} \mathrm{rad}$, considering the small gap.

Photography from transparent glass side is shown on Figure 5.

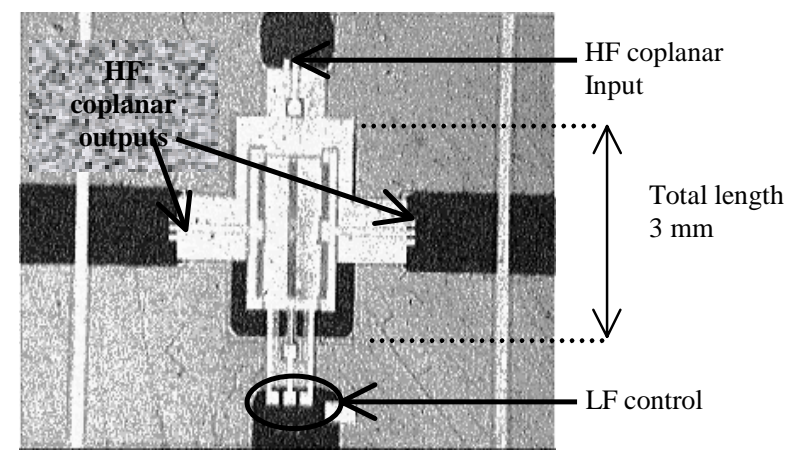

Figure 5. Top view of the prototype.

The technological process has been stabilised. We use SOI wafers $(500 \mu \mathrm{m}$ thick) to extract the torsional plate $(15 \mu \mathrm{m}$ thick) with deep plasma etching. Anodic bounding is necessary at the end of the process to create the capacitors. Seven photolithography levels have been used to complete this device.

\section{EXPERIMENTAL MEASUREMENTS}

Mechanical measurements of the moving silicon plate have been performed using an optical profilometer to control the geometry and an optical vibrometer to visualise resonance modes with interference fringes patterns, Figure 6 [5].

A longitudinal bend of the structure has been observed, as well as problems due to the torsional modes mixed with flexion modes.

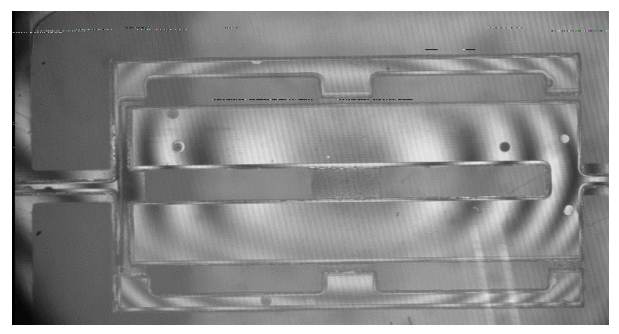

Figure 6. Resonance modes of the moving capacitors.

Microwave measurements (in the range 1-10 GHz) of the structure require special attention, because of very short transmission lines on silicon [6]. Coplanar waveguides are mostly used on the device, essentially located on the glass substrate. But a new kind of inverted microstrip lines (with silicon covering) and also microstrip lines directly on silicon have been studied.

The HF caracterisation is performed with different vector network analysers (HP8510C and HP8753D). For on-wafer MEM measurements [7], the workbench is equipped with a coplanar probe station: CascadeMicrotech probes. This measurement technique provides a calibration used to extend the reference planes to the probe tips end: the parameters extracted do not include those of the external connections and probes. However, during the on-wafer characterisation of the whole structure, parasitic phenomena associated with interconnect lines surrounding the plate may have an impact on measurements. Error sources deriving from wafer probing can be identified in two classes of signal degradations: losses and crosstalk. As the frequency is increasing, in our case 1 to $10 \mathrm{GHz}$, the influence of the small-signal access paths increases, thus limiting the measurement range, while adding their effects to the MEM device characterisation.

In order to avoid overwhelming of the S-parameters of the intrinsic MEM, and to determinate accurately the equivalent $\mathrm{HF}$ moving capacitors as well as the capacitance variation law $\mathrm{C}(\mathrm{V})$ versus the applied DC bias, we need to take into account the contribution of contact access and interconnect losses.

We propose to describe its contribution by a smallsignal high-frequency equivalent model in the $300 \mathrm{kHz}-$ 
$6 \mathrm{GHz}$ range. This model, presented on Figure 7, derived from the analysis of the parasitics structure.

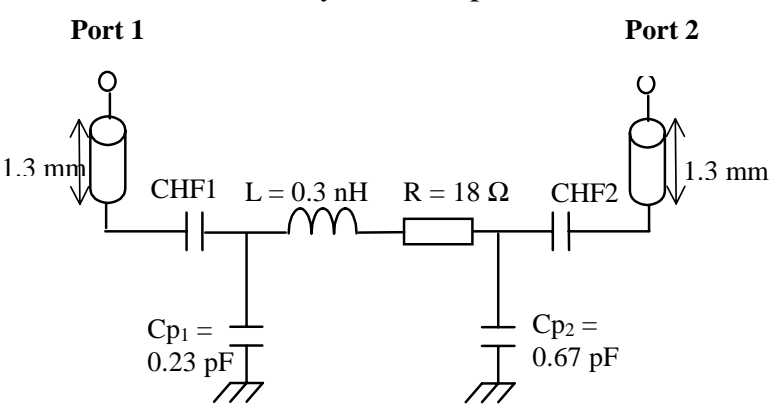

Figure 7. Equivalent electrical model of the plate.

Elements $\mathrm{CHF}_{1}$ and $\mathrm{CHF}_{2}$ represent the equivalent $\mathrm{HF}$ moving capacitors, while $\mathrm{Cp}_{1}$ and $\mathrm{Cp}_{2}$ the parasitic capacitors due to the oxide layer between silicon and gold. Capacitance measurements without DC bias give values for $\mathrm{CHF}_{1}$ and $\mathrm{CHF}_{2}$ in the range $0.5 \mathrm{pF}$ to $0.7 \mathrm{pF}$ for rectangle devices and $0.4 \mathrm{pF}$ to $0.5 \mathrm{pF}$ for square devices.

The connecting line of the two HF capacitors introduces a small inductive contribution (L) with a quite important resistive loss (R) essentially due to the poor quality of the $\mathrm{Cr} / \mathrm{Au}$ metallisations. The contact access and parasitic interconnect losses have also been represented with a line of $1.3 \mathrm{~mm}$ length.

The provided equivalent model is built using a Microwave Linear Simulator (HP-MDS). Optimisation routine is used to fit this equivalent circuit model to the measured S-parameters, see on Figures 8a \& 8b. The elements are optimised by minimizing the distance between the scattering elements measured by the network analyzer and those resulting from the simulation of the circuit on each frequency point in the $300 \mathrm{kHz}-6 \mathrm{GHz}$ range.

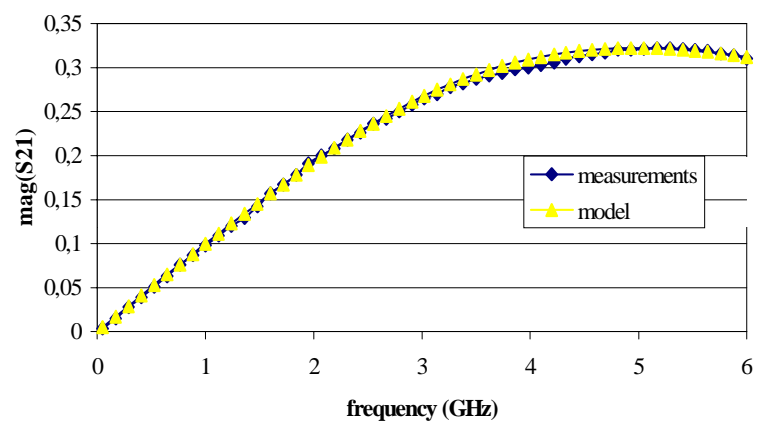

Figure 8a. Comparison between the circuit model and the S-parameter measurements for $\operatorname{mag}(\mathrm{S} 21)$.

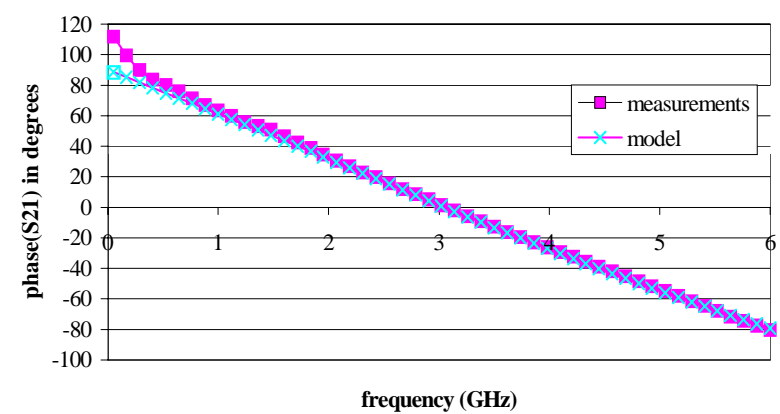

Figure 8b. Comparison between the circuit model and the Sparameter measurements for phase(S21).

Moreover, we tried to demonstrate the feasibility of the mixing function: DC polarisation on the right or left electrodes leads to opposite variations of the capacitor $\mathrm{CHF}_{1}$ (left) or $\mathrm{CHF}_{2}$ (right). These variations follow very well a square voltage law as expected for electrostatic actuation, see Figures 9 and 10.

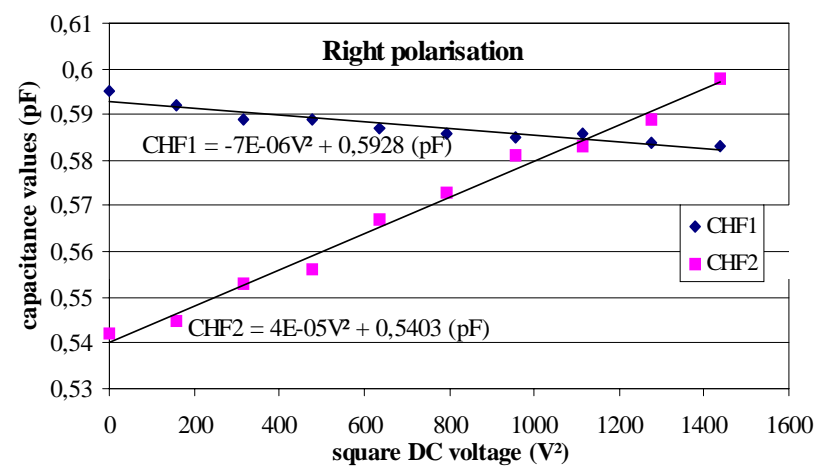

Figure 9. Law $\mathrm{C}\left(\mathrm{V}^{2}\right)$, on the right side.

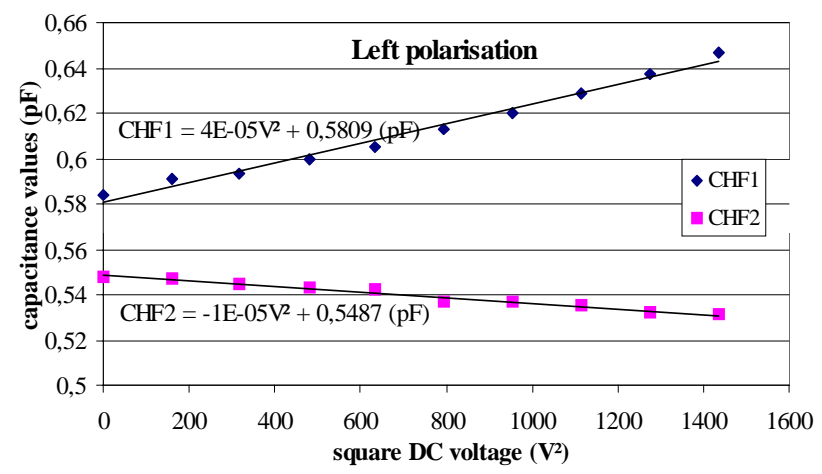

Figure 10. Law $\mathrm{C}\left(\mathrm{V}^{2}\right)$, on the left side.

Nevertheless, the above results also indicate a dissymetry of the capacitance change correlated with a combination of movements of flexion and rotation. These results are in accordance with mechanical measurements showing a preponderant flexion mode, hiding the rotation mode: in fact, the rotation only contributes up to $20 \%$ to the capacitance variations, because of a strong torsion rigidity of the arms. 
Moreover, we also performed modulation measurements still with on-wafer probing to verify the feasibility of the micro-modulator. The experimental set-up is illustrated on Figure 11: while we control the electrodes alternaly with a low frequency generator, as presented on Figure 12, we apply the HF signal at $1 \mathrm{GHz}$ using a ground-signal-ground configuration for the probes (pitch $150 \mu \mathrm{m}$ ), and we collect the different tones generated by the LF modulation of the capacitances CHF1 and CHF2 on a spectrum analyser.

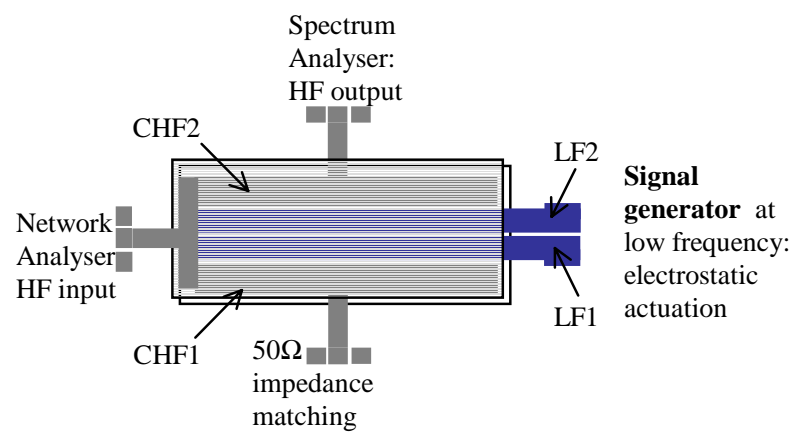

Figure 11. Experımental set-up for AC control.

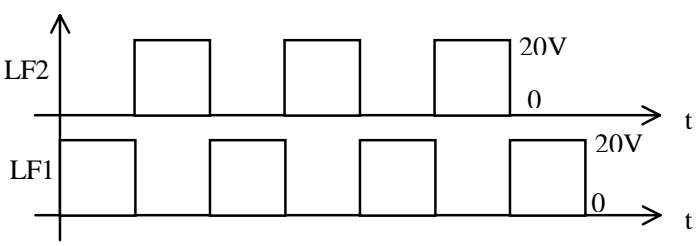

Figure 12. Chronograms of the low frequency control (LF1 \& LF2).

We tested the LF modulation from $200 \mathrm{~Hz}$ up to $12 \mathrm{kHz}$, keeping the HF input at $1 \mathrm{GHz}$, Figure 13. We esssentially detected odd tones (P1, P3, P5) in the output spectrum: we collected the required shifted frequency illustrating the micro-modulator principle, but also the fundamental carrier (P0) at $1 \mathrm{GHz}$ because the experimental set-up is not yet available for differential measurements. A low modulation index is observed (40 dB between P0 and P1), due to the weak variations of the moving capacitors. And we can observe a cut-off frequency aroung $6 \mathrm{kHz}$.

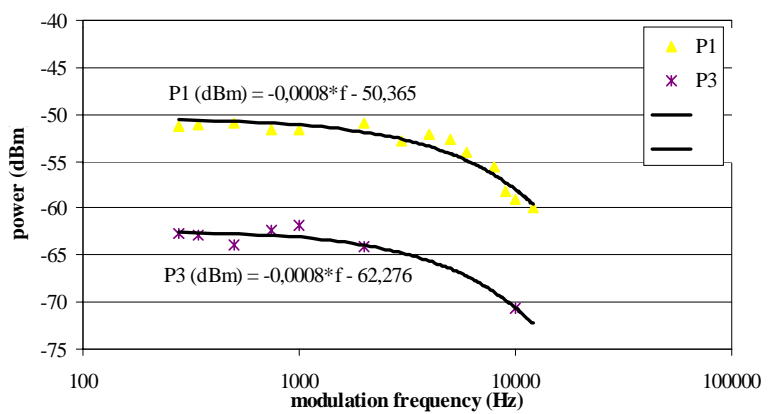

Figure 13. Power measurements P1 (tone 1) and P3 (tone 3) with carrier@1 GHz.
We are currently developing another equivalent circuit model, based on the first one, which considers the experimental set-up implementation and constraints in the modulation characterisation mode.

\section{CONCLUSION}

MEMS including microwave functions and integrated on silicon can be very interesting for future applications in autonomous systems. The principle of moving capacitors can also be useful for new filtering techniques. The paper describes a silicon micro-device used as a passive frequency shifter. The feasibility of the micro-mechanical capacitors is demonstrated, as well as the mixing principle function. The important result concerns the electrical model giving the capacitor law versus the DC bias in connection with microwave measurements.

Some improvements will be further considered to increase performances of the device, especially to reduce attenuation on the microwave transmission lines on the silicon plate, and to increase the resonance frequency of the device.

Acknowledgements - This work was performed inside the $\mathrm{PFM}^{1}$ with the help of P. Sangouard for technological developments at ESIEE, and A. Bosseboeuf for mechanical measurements at IEF.

\section{REFERENCES}

[1] Villegas M., Bildstein P., Nicole P., Translateur de fréquence micro-ondes pour capteur intelligent autonome, Proc. of JNM, p. 270-271, 1997.

[2] Bazin G., Sangouard P., Bildstein P., Design of a micro-mechanical capacitor with microwave functions, EPJ Applied Physics, vol.9, p. 75-80, 2000.

[3] PetERSEN K. E., Silicon torsional scanning mirror, IBM Journal of Research and Development, vol.24, p. 631, 1980.

[4] JUDY J. W., MÛLLER R. S., Magnetic microactuation of torsional polysilicon structures, Sensors and Actuators, vol. 53, p. 392-397, 1996.

[5] Bosseboeuf A., Thouvenin J.L., Quemper J.M., White light interferometric profilometry, a powerful technique for the control micromechanical devices deformations and fabrication, Proc. of MME'99, p. 59-62, 1999.

[6] PonchaK G. E., RF transmission lines on silicon substrates. Proc. of $29^{\text {th }}$ European Microwave Week, p. 158-161, 1999.

[7] JOHN, T. and al., Advancements in on-wafer probing calibration techniques, Proc. HP technical papers, RF/Microwave and Lightwave Symposium and Exhibition, 1991.

\footnotetext{
${ }^{1}$ Pôle Francilien Microsystèmes
} 